\title{
Innovación y reinvención en la distancia
}

\section{Innovation and reinvention in the distance}

\author{
Anabel Mariela González \\ https://orcid.org/0000-0002-6121-8754 \\ anabelmargon24@gmail.com \\ Facultad de Odontología | Universidad \\ Nacional de La Plata | Argentina
}

\author{
Patricia Susana Pérez \\ https://orcid.org/0000-0001-7216-722X \\ patogelptyg@gmail.com \\ Facultad de Odontología | Universidad \\ Nacional de La Plata | Argentina
}

\author{
Adrián Alejandro Abal \\ https://orcid.org/0000-0003-4356-9961 \\ drpanacea2003@yahoo.com.ar \\ Facultad de Odontología | Universidad \\ Nacional de La Plata | Argentina
}

\author{
Melina Procopio Rodríguez \\ https://orcid.org/0000-0002-2732-5302 \\ melina.p.r@hotmail.com \\ Facultad de Odontología | Universidad \\ Nacional de La Plata | Argentina
}

\author{
Andrea Tanevitch \\ https://orcid.org/0000-0002-7797-7659 \\ atanevitch@yahoo.com.ar \\ Facultad de Odontología | Universidad \\ Nacional de La Plata | Argentina
}

\section{RESUMEN}

El objetivo de este trabajo es mostrar el camino de reinvención que tomó nuestro grupo de trabajo docente. Describiremos estrategias que pudimos poner en práctica, con el fin de mejorar y/o mantener la calidad en el proceso de enseñanza y aprendizaje, dentro del actual contexto de emergencia sanitaria global. En pos de garantizar la continuidad pedagógica de los y las estudiantes, como consecuencia de la pandemia que nos ha atravesado a lo largo de este año, nos vimos obligados a adecuar nuestra labor docente y mantener el calendario académico. Mediante el uso de medios digitales logramos cubrir necesidades académicas, pedagógicas, administrativas y personales de nuestro alumnado ante la inesperada situación. El hecho de poder acceder a cursos de capacitación docente en la modalidad virtual y de carácter intensivo para el uso de TIC, nos abrió un abanico de posibilidades impensadas que nos marcó el camino para transitar nuevas actividades enriquecedoras con el fin de hacerle frente al nuevo paradigma vigente. Nuestro desempeño docente corresponde a las asignaturas pertenecientes a los primeros años de la carrera que se encuentran bajo la articulación del Departamento de Ciencias Biológicas Básicas y Aplicadas y no requieren presencia en el área clínica.

PALABRAS CLAVE

Reinvención, Docencia, TIC 
KEY WORDS

Reinvention, Teaching, ICT.

\section{SUMMARY}

The objective of this work is to show the path of reinvention that our teaching work group took. We will describe strategies that we were able to put into practice, in order to improve and / or maintain quality in the teaching and learning process, within the current context of a global health emergency. In order to guarantee the pedagogical continuity of the students, as a consequence of the pandemic that has crossed us throughout this year, we were forced to adapt our teaching work and maintain the academic calendar. Through the use of digital media we are able to meet the academic, pedagogical, administrative and personal needs of our students in the face of the unexpected situation. The fact of being able to access teacher training courses in the virtual modality and of an intensive nature for the use of ICT, opened up a range of unthinkable possibilities that marked the way for us to go through new enriching activities in order to face the new paradigm valid. Our teaching performance corresponds to the subjects belonging to the first years of the career that are under the articulation of the Department of Basic and Applied Biological Sciences and do not require presence in the clinical area. 


\section{INTRODUCCIÓN}

A partir de mediados de marzo de 2020 la COVID-19 fue declarada pandemia según la OMS y a partir del registro de casos positivos en nuestro país se tomaron medidas de acción por el gobierno nacional. La medida de mayor impacto fue el aislamiento preventivo, social y obligatorio (APSO) que modificó la realidad de todos los argentinos en distintos ámbitos. En las universidades y considerando la autarquía propia de las casas de altos estudios, la Universidad Nacional de La Plata (UNLP) ratificó su calendario y determina sobre la necesidad de mantener la continuidad pedagógica en todas sus Facultades (UNLP, 2020). El día 19 de marzo, en el Boletín Informativo semanal de Educación a Distancia (UNLP) se publica un comunicado, el cual expresa la interrupción del dictado de clases presenciales por un lapso de 12 días, adecuándose a las resoluciones emanadas del Ministerio de Educación y Salud de la Nación. Junto con el mismo, la siguiente notificación: "Diseñamos en tiempo récord un Programa de apoyo a la Educación a Distancia para Facultades y Colegios en el contexto de la epidemia COVID-19. Todos los docentes podrán implementar mecanismos de comunicación en línea con los estudiantes a través de las aulas virtuales de las plataformas propias de cada Facultad o Colegio, o en el entorno Aulasweb" (PAED-UNLP, 2020). Dicho plan de apoyo sigue en marcha hasta la fecha y se ha ido adaptando a las necesidades que fueron surgiendo.

\section{DESARROLLO}

En el marco del decreto de la emergencia sanitaria por la pandemia de Covid-19, en la Facultad de Odontología, se implementaron con celeridad, estrategias de continuidad educativa conllevando a las asignaturas a elaborar un plan de contingencia adecuado al lineamiento 
institucional para desarrollar el ciclo lectivo vigente. En primera instancia siguiendo las recomendaciones expresadas por Fainholc (2013), creímos que era prioridad indispensable para el docente desarrollar competencias que incluyan el manejo de los entornos virtuales para la educación a distancia, incorporando el uso de las herramientas que ésta nos brinda. Se repensó el escenario de formación y las posibilidades que se presentaban en medio de la incertidumbre imperante. Nos planteamos distintos interrogantes. ¿Se pueden enseñar los contenidos de este campo sin "ir a la Facultad"? ¿Se pueden hacer prácticas en este tiempo de excepcionalidad? ¿Las prácticas serán las mismas? ¿Se puede mantener el contacto con el alumnado?

Tomando como premisa objetivos de la Dirección General de Educación a Distancia y Tecnologías (UNLP) comenzamos a desarrollar soportes y entornos necesarios para la realización de diversas actividades académicas. Además de gestionar propuestas de actividades y programas educativos utilizando la modalidad no presencial, incorporamos el uso de las tecnologías de la información y la comunicación en las propuestas formativas de la modalidad a distancia. A partir de ello asumimos el desafío, de sostener nuestra capacitación docente mediante la formación continua brindada por el "Programa de Apoyo a la Educación a Distancia" (PAED) de la Universidad Nacional de La Plata, especialmente diseñado para esta contingencia. En este sentido, los webinar para la capacitación de los docentes elaborados sumado a los cursos cortos a distancia resultaron pilares para la adquisición y fortalecimiento de habilidades tecnológicas con perspectiva pedagógica.

\section{Una estrategia fundamental es el conocimiento de las situa- ciones particulares de los y las estudiantes, estableciendo pre- viamente sus potencialidades y debilidades y de esta manera, formular distintas propuestas de enseñanza y aprendizaje que logren atender la diversidad y desigualdades emergentes}

Una estrategia fundamental es el conocimiento de las situaciones particulares de los y las estudiantes, estableciendo previamente sus potencialidades y debilidades y de esta manera, formular distintas propuestas de enseñanza y aprendizaje que logren atender la diversidad y desigualdades emergentes. En este sentido, compartiendo las reflexiones de Maggio (2020) es importante mantenerse flexibles y darse la posibilidad de adaptar los contenidos programáticos en función de las condiciones del contexto de los y las estudiantes. Para comprender estas circunstancias, los instrumentos como las encuestas anónimas y voluntarias nos proveen de los datos necesarios para dirigir la metodología más adecuada. 
Nuestro desempeño docente corresponde a las asignaturas pertenecientes a los primeros años de la carrera que se encuentran bajo la articulación del Departamento de Ciencias Biológicas Básicas y Aplicadas y no requieren prácticas en el área clínica, por lo que el desarrollo virtual de los contenidos teóricos y prácticos permitió que los y las estudiantes accedan a la condición final del curso cumplimentando los requisitos de cada instancia.

\section{DISEÑO DE AULAS VIRTUALES Y PRODUCCIÓN DE CONTENIDOS DIGITALES}

Según Pérez Serrano (2015) la inclusión de la educación permanente, se ha convertido no sólo en exigencia, sino en necesidad ineludible y un factor decisivo para la sociedad del conocimiento y la cultura, para igualar las oportunidades y propiciar la cohesión social, para construir comunidad, y para generar calidad de vida.

En el momento de asumir la transformación de nuestras clases decidimos adquirir competencias para utilizar nuevos recursos y herramientas que nos sirvieran para ir adaptando nuestras tradicionales a la modalidad virtual. Además, contar con una plataforma institucional (MOODLE), facilitó la accesibilidad de la gran mayoría del estudiantado y se pudo mantener la comunicación sobre la secuencia de contenidos, actividades, tareas y tiempos de resolución de las mismas. Organizando de este modo el cronograma del cuatrimestre, pudimos ir incorporando diversos recursos que brinda la plataforma como el uso del chat, los foros, el correo interno, que posibilitaron el intercambio y el contacto entre estudiantes y entre estudiantes y docentes. El uso de las TIC en el universo del estudiante, favoreció la utilización de contenidos digitalizados como forma de transmitir información y en razón de ello, se elaboró material didáctico en formato PowerPoint con audio y sin audio, videos, material de lectura, Trabajos prácticos virtuales, de acuerdo a los contenidos del programa de estudio. Además, se recomendó el uso de la biblioteca virtual presente en la plataforma.

La plataforma Moodle es un entorno muy versátil para desarrollar aprendizajes constructivistas y colaborativos pero también permite la presentación de archivos estáticos, documentos, videos mediante las funciones de integración de medios para entregar contenido a los alumnos. Con la herramienta tareas, las actividades prácticas se realizaron con frecuencia semanal. La construcción de las pautas de resolución y entrega de las mismas significó un aprendizaje para docentes y estudiantes, utilizando expresiones lo más detalladas posibles como indicar el modo de presentación, fecha de entrega y tiempo de resolución. La devolución de las tareas y la retroalimentación fue realizada tanto en forma asincrónica mediante la plataforma 
Moodle como en forma sincrónica en las reuniones de Zoom. De esta forma, se favoreció la estrategia de evaluación formativa para un mejor seguimiento del aprendizaje y adquisición de saberes.

\section{MANTENIMIENTO DE LA ACTIVIDAD PRÁCTICA Y DISTINTAS ESTRATEGIAS DE EVALUACIÓN}

En coincidencia con González (2016) creemos que fomentar el trabajo colaborativo y en línea es un pilar para los nuevos tiempos, donde los jóvenes se relacionan a través de redes sociales informatizadas.

Durante la incorporación de nuevas herramientas y diseño de distintas estrategias fue necesario poder gestionar tiempos, elaborar consignas que fueran entendidas y consensuadas y a su vez generar un proceso de evaluación formativa. Las problemáticas que se presentaron en el día a día, nos encontró ante la necesidad de lograr el acompañamiento de los estudiantes. La posibilidad de acceder al curso "Enseñar en la distancia: Tutorías y estrategias de enseñanza mediadas por tecnologías" (CAVILA-UNLP), nos capacitó sobre la importancia de transformar la planificación de la presencialidad a la virtualidad. Como sostiene Martínez y Briones (2007), la tutoría puede ser entendida como "una relación de ayuda" que puede adquirir distintas modalidades según el modelo predominante individualizado, en grupo, entre iguales, de asignatura. No obstante, el aislamiento y la educación a distancia, resignifican la multifuncionalidad de la tutoría como guía para el aprendizaje, la filiación académica y la contención socio afectiva. La falta de contacto directo genera un vacío, donde descubrimos que era fundamental canales de comunicación sincrónica que pudieran llevar a mejorar la retroalimentación inmediata y el intercambio socioeducativo. Una estrategia interesante es la "escalera de retroalimentación" porque permite poner en valor el trabajo realizado en función del proceso desarrollado por los y las estudiantes mediante una retroalimentación positiva y no como una serie de acciones punitivas (Anijovich, 2014). De allí que los primeros canales de comunicación disponibles al inicio del aislamiento, fueron multiplicándose en espacios de interacción asincrónica y sincrónica. El potencial de comunicación que provee la plataforma fue reforzado por las publicaciones del centro de estudiantes mediante las redes sociales como su página de Instagram, constituyéndose en la vía de comunicación permanente, ante la posibilidad de algún imprevisto debido a su alcance masivo.

Fuimos adquiriendo habilidades a medida que avanzaba la cursada acerca del trabajo colaborativo, la coevaluación de pares y de esta forma los trabajos en grupo pudieron ser resueltos a través de foros, uso del Drive y reuniones sincrónicas presenciales de videoconferencias mediante la plataforma Zoom. 
Durante la incorporación de nuevas herramientas y diseño de distintas estrategias fue necesario poder gestionar tiempos, elaborar consignas que fueran entendidas y consensuadas y a su vez generar un proceso de evaluación formativa. Las problemáticas que se presentaron en el día a día, nos encontró ante la necesidad de lograr el acompañamiento de los estudiantes.

Dentro de este contexto, el rol tutorial del docente debió ser asumido reforzando el vínculo docente-alumno, en donde el uso de redes sociales fue fundamental, siendo una de las más elegidas el Whatsapp, dado que el mismo no genera gasto de datos de internet a la vez que brinda un buen nivel de accesibilidad y facilita la posibilidad de formar grupos entre pares que enriquecieron el trabajo colaborativo. El uso de foros como herramienta de diálogo, logró resumir en los debates en grupo, los aportes de los estudiantes.

Dentro de este contexto, el rol tutorial del docente debió ser asumido reforzando el vínculo docente-alumno, en donde el uso de redes sociales fue fundamental, siendo una de las más elegidas el Whatsapp, dado que el mismo no genera gasto de datos de internet a la vez que brinda un buen nivel de accesibilidad y facilita la posibilidad de formar grupos entre pares que enriquecieron el trabajo colaborativo. El uso de foros como herramienta de diálogo, logró resumir en los debates en grupo, los aportes de los estudiantes.

A la hora de la evaluación se utilizaron distintas estrategias, siguiendo la línea de establecer una evaluación continua y permanente se pudieron incorporar, como material didáctico, los videos interactivos como propuesta de autoevaluación junto con los cuestionarios. Para la acreditación de los cursos en la instancia evaluadora, se implementaron parciales intermedios, integradores y exámenes finales mediante cuestionarios de la plataforma Moodle. Además se hicieron valoraciones globales e individuales de las actividades realizadas durante los encuentros sincrónicos.

EL DESAFÍO DE MANTENERNOS EN CONTACTO A PESAR LA CONTINGENCIA QUE VIVIMOS

Con el fin de sostener la personalización y ubicar el aprendizaje en un lugar donde el estudiante logre su sentido de pertenencia al lugar 
manteniendo su identidad, se logró mantener el contacto con los y las estudiantes a través de distintas redes, teniendo en cuenta como afirman Tourn y Berruhet (2015), las generaciones de estudiantes se están educando y vinculando a través de los espacios que ofrece Internet como agente socializador, proceso que es acompañado por dos aspectos complementarios: la adhesión al ciberespacio y la revolución digital. El uso de las redes sociales por los y las jóvenes, supone nuevas formas de relacionarse con los otros y con uno mismo. La importancia de la "red" radica en el lugar que los sujetos le asignan a los vínculos, la interacción, la imagen y el intercambio dinámico entre los mismos, representando así, la dimensión sociocultural de las TIC. Como partícipes de la sociedad del conocimiento en este momento singular y excepcional nos ha ubicado en un tiempo de reflexión que implica un cambio en la cultura docente.

El protagonismo que ha tomado la educación a distancia en tiempos de COVID en palabras de Decoud de Canale (2020) "implica un cambio en la cultura docente, un paradigma que se centra en el aprendizaje del estudiante, y requiere de nuevas metodologías de enseñanza que fomenten el autoaprendizaje, colaboración, una comunicación constante e interacción entre docente y equipo de estudiantes, el seguimiento al proceso y progreso de las actividades y logros. La interacción entre los distintos elementos que configuran el entorno virtual, como los contenidos, medios de comunicación y actores, son esenciales en la modalidad virtual".

\section{CONCLUSIÓN}

El desafío de reinventar el aula, las estrategias de enseñanza, los materiales didácticos, en el marco de la imprevisibilidad y los cambios globales que impactan en la práctica pedagógica, nos permite reflexionar sobre la necesidad de la formación docente continua, la importancia de generar comunidades a distancia con sentido filiatorio, capaces de interactuar desarrollando habilidades comunicativas orales y escritas mediadas por tecnología. La emergencia sanitaria nos movilizó desde nuestra zona de confort hacia una experiencia nueva e incierta para muchos de nosotros, pero que con aprendizaje y acompañamiento pudimos consolidar esta modalidad de educación virtual, impensada antes del aislamiento preventivo, social y obligatorio. Estas transformaciones se constituyeron en innovaciones que no sólo permitieron transitar estos escenarios complejos de la pandemia, sino que adquieren relevancia como una práctica docente permanente aun en el aula presencial. 


\section{BIBLIOGRAFÍA}

Decoud de Canale Carla (2020). "Protagonismo de la educación a distancia en tiempos de COVID-19. Un reto que empuja hacia el cambio de paradigma". Ciencia y Tecnología Politécnica UNA. Recuperado de: https://cytpolitecnicauna.blogspot.com/2020/06/protagonismo-de-la-educacion-distancia.html

Fainholc, B., Nervi, H., Romero, R., \& Halal, C. (2015). “La formación del profesorado y el uso pedagógico de las TIC". Revista De Educación a Distancia (RED), (38). Recuperado a partir de https://revistas.um.es/red/article/view/234081

González, A. H. (2016). "Los jóvenes, las TIC, y primer año en la Universidad". Trayectorias Universitarias, 2(3). Recuperado de: https://revistas.unlp.edu.ar/ TrayectoriasUniversitarias/article/view/3023

Maggio, M. (2020). "Reinventar la clase en tiempos de pandemia. 3ras. Jornadas sobre Las Prácticas Docentes en la Universidad Pública". La Plata, junio.

Martínez, M. T., Briones S. M. (2007). "Contigo en la distancia: la práctica tutorial en entornos formativos virtuales". Pixel-Bit. Revista de Medios y Educación (29), 81-86 Recuperado de: https://www.redalyc.org/articulo.oa?id=36802907

Programa de apoyo a la Educación a Distancia para Facultades y Colegios en el contexto de la pandemia de COVID-19. PAED (2020) Dirección de Educación a Distancia Universidad nacional de La Plata.

Pérez Serrano, M. G. (2001). "Aprender a lo largo de la vida. Desafío de la sociedad actual". Localización: Agora digital, 1. Recuperado de: http://rabida.uhu. es/dspace/bitstream/handle/10272/3435/b1575957x.pdf?sequence=1

Tourn, L.; Berruhet, M. J. (2015). “Los vínculos en las nuevas juventudes + El poder de la imagen: red social Instagram. Qué buen momento...." III Jornadas de TIC e Innovación en el Aula, La Plata, Recuperado de: http://sedici.unlp.edu. ar/handle/10915/48699 